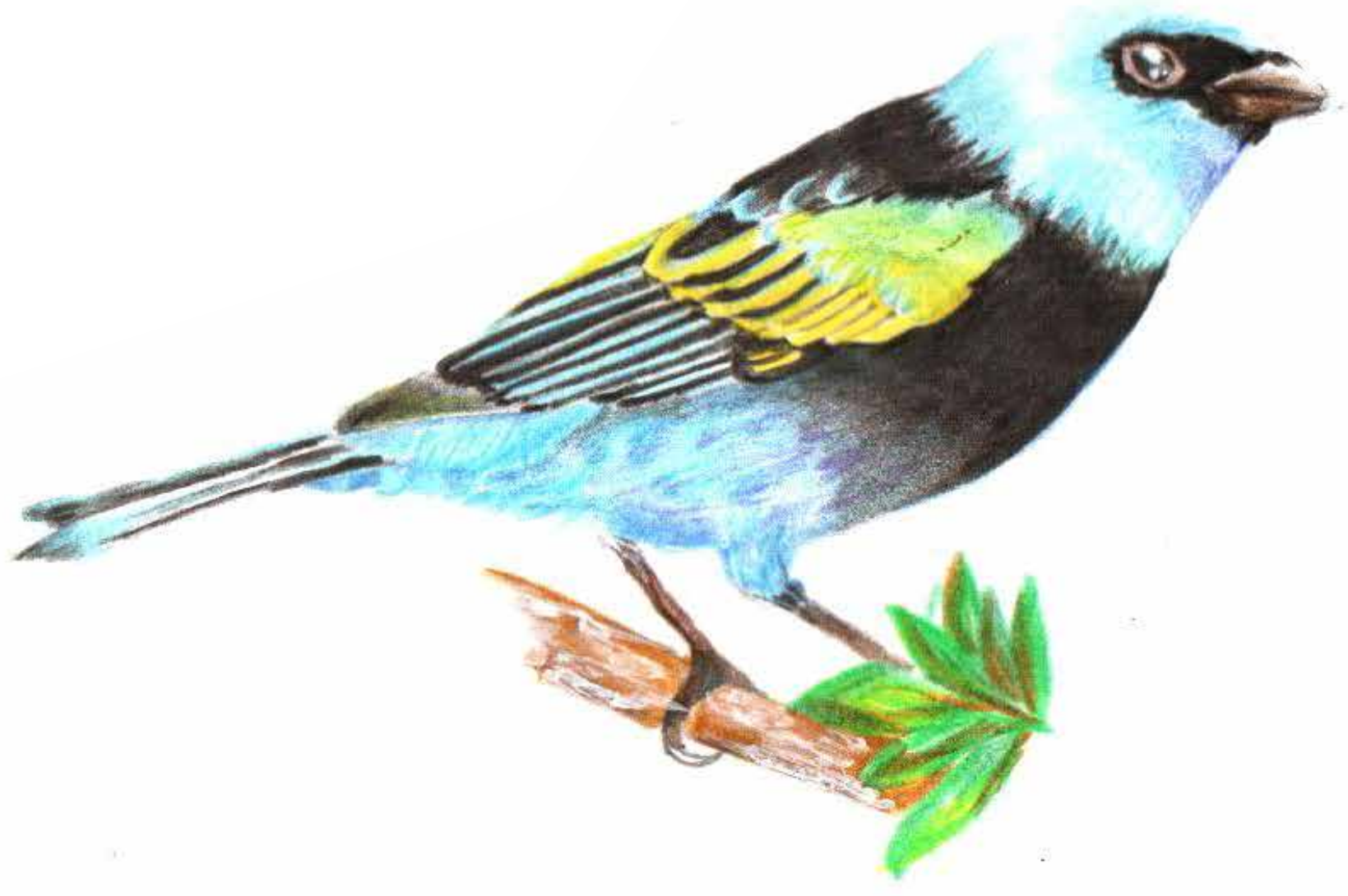

Jeny Andrea Fuentes Acevedo

Estudiante de Sexto Semestre de Licenciatura en Biología

Universidad Pedagógica Nacional

Técnica: Lápices de colores 


\title{
RENOVACIÓN DE MEMORIA HISTÓRICA SOBRE LA CULTURA DEL MAÍZ CON LA COMUNIDAD EDUCATIVA IED EL CARMEN SEDE SAN FRANCISCO MUNICIPIO DE GUASCA
}

\section{RENEWAL OF REMEMBRANCE ON CORN CULTURE COMMUNITY EDUCATION IED EL CARMEN SAN FRANCISCO HEADQUARTERS, COUNTY GUASCA}

\author{
Eliana Marcela Reinoso Sabogal ${ }^{1}$
}

\section{Resumen}

En este escrito se muestra un ejercicio de renovación de memoria sobre usos y significados culturales del maíz a partir del trabajo con la comunidad educativa de los grados cuarto y quinto de la IED El Carmen sede San Francisco. Este se realizó desde un enfoque cualitativo trazando una ruta metodológica que incluye técnicas e instrumentos como el diario de campo, entrevistas, conversaciones, fotografías y grabaciones.

Con la información obtenida se elaboró una matriz a partir del análisis de resultados, que los estudiantes poseen una memoria biocultural ${ }^{2}$, unos saberes trasmitidos porvía oral de generación en generación, siendo posible la memoria individual y colectiva. Estos procesos representan una forma de enseñar biología y su relación con lo vivo desde un enfoque intercultural en el cual cada persona interactúa constantemente con el otro desde su conversar.

\section{Palabras clave}

memoria histórica, memoria biocultural, maíz, interculturalidad, enseñanza de la biología

\section{Abstract}

In this paper, we show an exercise in renewal report on uses and cultural meanings of corn from the educational community work courses IED 4 and 5 of the San Franciscobased Carmen. This was done from a qualitative approach drawing a methodological route includes techniques and instruments such as field notes, interviews, conversations, photographs and recordings.

With the information obtained matrix was performed taking as analysis results, students have a biocultural memory, a knowledge transmitted orally from generation to generation, with the possibility of individual and collective memory. These processes represent a way of teaching biology and its relation to the living from an intercultural approach in which each person is constantly interacting with each other from their conversation.

\section{Keywords}

historical memory, biocultural memory, corn, multiculturalism, teaching biology

1 Estudiante Licenciatura en Biología, Universidad Pedagógica Nacional. Artículo producto de investigación realizada en la escuela rural San Francisco en el municipio de Guasca (Cundinamarca). Correo electrónico: marcela-1530@hotmail.com

2 Memoria biocultural: es la interacción de miles de años entre las culturas y sus ambientes naturales, estas conforman un complejo biológico/cultural. Este se da desde todos los conocimientos sobre la naturaleza, que conforman expresiones de una cultura en donde se refleja las observaciones sobre el entorno que son mantenidas, trasmitidas y perfeccionadas a través de largos periodos, sin las cuales la supervivencia de los grupos humanos no hubiera sido posible. Se trata de los saberes, transmitidos oralmente de generación en generación y en especial aquellos conocimientos imprescindibles y cruciales, por medio de los cuales la especie humana fue moldeando sus relaciones con la naturaleza (Toledo y Barrera, 2008, 25). 


\section{Introducción}

Guasca es un municipio con mucha historia. En este lugar habitaron los Muiscas que dejaron leyendas, mitos y algunas tradiciones que se fueron perdiendo con el paso de los años.

Para la comunidad educativa es su casa, es donde siembran, esto forma parte de su vida; pero algunas de estas prácticas y costumbres se están perdiendo, casi no se siembra para el consumo sino para comerciar; los abuelos ya no tienen a quién enseñar a tejer, como cuenta la señora Graciela, abuela de una estudiante del colegio. Sus manos ya están muy maltratadas por el trabajo, y su nieta es muy pequeña para enseñarle, además comenta que los muchachos ya no saben qué es una ruana, porque usan chaquetas.

Otra de las prácticas que se están desapareciendo es la siembra de maíz. Las abuelas recuerdan que antes en sus casas tenían sembrado, hoy son muy pocas las personas que lo hacen, tal vez porque se dedicaron a cosechar papa y otros alimentos para vender. Otros comentan que se demora mucho para dar cosecha y además se llenan de hongos sino se fumiga. Precisamente, por las dinámicas actuales de colonización y globalización, ahora sus esfuerzos de siembra están dirigidos a productos comercializables, de corto ciclo de producción y de altos cuidados con fungicidas y abonos inorgánicos.

Estas son algunas de las transformaciones que se vienen presentando en las prácticas culturales de los habitantes de Guasca, las cuales los alejan profundamente de aquello, que, para culturas como la zenú ${ }^{3}$, representa sus orígenes; sembrar maíz es sembrarse a sí mismos, conocimiento que también comparten con el pueblo inga ${ }^{4}$. Dichas prácticas fortalecen su identidad cultural y el sistema de organización social desde los procesos del cultivo del maíz, para ellos es algo más que una simple semilla, es espíritu, es alma, es vida.

Otro ejemplo lo podemos tomar del Perú, en donde el proceso de la utilización del maíz para preparar la chicha es parte de su cultura; utilizar ollas de barro y otros elementos enriquecen esta cultura del maíz, la utilidad de esta bebida depende de la ocasión, que tiene además una historia desde los pueblos ancestrales de Indoamérica.

3 El pueblo indígena zenú se localiza en los resguardos de San Andrés de Sotavento en el departamento de Córdoba, y EI Volao, en Urabá. Además en varios asentamientos pequeños en Sucre, Antioquia y Chocó.

4 El pueblo inga habita principalmente en el Valle del Sibundoy -a 2200 metros sobre el nivel del mar- en el departamento del Putumayo. Son descendientes de los incas y arribaron a la región como avanzadas militares en el proceso de expansión del imperio.
En Colombia, el maíz, su siembra y demás rituales provienen de una cultura ancestral y popular. Montes y Rodríguez (1975), en su recopilación de historias y prácticas asociadas al maíz, muestran una colección de coplas, paremias, adivinanzas en las que se evidencian algunas utilidades y la importancia de este producto, entre ellas: medicina popular, gastronomía, creencias, costumbres y juegos. Esta es una de las razones para renovar memoria en la comunidad educativa, pues de esta manera es posible retomar las tradiciones que los abuelos de los estudiantes poseen y que son trasmitidas por la oralidad y desde sus rituales. Solo así estarían en capacidad de apropiarse de su pasado y de reafirmar la identidad cultural de su pueblo.

Una habitante de la región y madre de familia comenta: "Se han perdido las tradiciones culturales por que las personas ya no viven acá y porque ahora todo se compra hecho, aunque algunos hacen las arepas" (Fabio Sánchez, diario de campo, 3 de septiembre, 2012). Hay un desplazamiento desde Guasca hacia otras regiones por distintas razones probablemente falta de empleo, pérdida de cultivos, entre otros y desde otras regiones hacia Guasca, esta es una de las posibles razones de la pérdida de las prácticas y costumbres, como el cultivo del maíz, realizadas en esta región.

Pero esta situación no es particular de Guasca, Lloréns (2008) afirma en sus investigaciones que países como el Perú, la cultura asociada al maíz, se está refundiendo al cambiar sus prácticas en torno a este. Por ejemplo, en la actualidad ya no usan ollas de barro para las preparaciones, sino recipientes de aluminio; anteriormente había expendios de chicha llamados "chicherías", pero han desaparecido.

La pérdida de estas prácticas implica un olvido de la identidad cultual de la comunidad de Guasca, que los caracteriza por los procesos empleados a través de la historia para sus cultivos, costumbres y tradiciones, basados en los conocimientos de las comunidades locales que fueron trasmitidos por generaciones. Por otro lado en el contexto global se ve amenazada por fenómenos de la modernidad como son los procesos técnicos y económicos, además de los informáticos, sociales y políticos, que los aleja cada día más de las costumbres, tradiciones, prácticas, pensamientos y sentires.

Durante los últimos 200 años, la chicha ha ido perdiendo sus vínculos con el ambiente; anteriormente en el Perú todo estaba atado de forma orgánica, al mismo tiempo la producción local de chicha ha dejado de lado sus antiguas conexiones con el medio que la rodeaba y con ello han disminuido sus virtudes como producto. 
En nuestro país, en cambio, hay un interés por parte de las alcaldías y medios de comunicación por incentivar y difundir la participación en eventos culturales regionales como ferias, fiestas y proyectos educativos, con el fin de recordar las tradiciones y aprender cada día de la diversidad cultural y étnica del territorio.

Desde aquí la importancia del enfoque intercultural, de desempolvar las tradiciones y conocimientos ancestrales para darles vigencia y otra posibilidad de existencia; por esta razón surge el propósito que los estudiantes de la comunidad educativa El Carmen sede San Francisco de los grados cuarto y quinto, identifiquen prácticas entorno al maíz e indaguen historias asociadas y comprendan su significado cultural, para renovar la memoria histórica.

\section{Metodología}

Este trabajo se desarrolla con un enfoque de investigación cualitativo, entendida esta última como una actividad localizada en un lugar y tiempo determinados, que sitúan al investigador en el mundo. Consiste en una serie de prácticas interpretativas y materiales que hacen al mundo visible. Estas acciones transforman el mundo, lo convierten en una serie de representaciones (Denzin, Lincoln y Vonna, 2005, pp. 1). Se utilizan técnicas e instrumentos como notas de campo, entrevistas, conversaciones, fotografías y grabaciones. En este grado, la investigación cualitativa implica un acercamiento interpretativo y naturalista del mundo. En consecuencia, el investigador estudia los objetos y los sujetos en sus escenarios naturales, intentando dar sentido o interpretar los fenómenos en términos de los significados que las personas les dan y en conversación con los referentes académicos.

Se utilizan técnicas o estrategias como: a) la observación participante, que implica el análisis y síntesis de una situación en particular; b) el cuaderno de campo, que ha sido de gran importancia durante la planeación del proyecto y tendrá validez en los resultados de esta investigación, ya que en este, se registran las experiencias vividas y los hechos observados para luego sistematizar los datos; c) los conversatorios, en los que se da un espacio para hablar con el otro; d) las visitas realizadas a las casa de los familiares de los estudiantes, durante los recorridos por el territorio para preguntar sobre la cultura del maíz, y e) el registro audiovisual para evidenciar el proceso de la investigación.

Después de haber escuchado, observado y tomado nota de todas las experiencias de vida de los estudiantes, la forma en que viven, sus costumbres, sus juegos, hasta su forma de expresarse durante el trabajo de campo, se pudo identificar que ellos no reconocen sus tradiciones ni forman parte de las costumbres familiares; estas han ido desapareciendo (por ejemplo, usar ruana). Así mismo los padres reconocen la importancia de enseñarles a sus hijos a sembrar con técnicas tradicionales para mantener este conocimiento de generación en generación. Se tuvo en cuenta esta contextualización, los sentires de los estudiantes y de la comunidad en general, y es allí cuando surge el siguiente cuestionamiento: ¿Cómo renovar memoria sobre usos y significados del maíz en la comunidad educativa de El Carmen sede San Francisco?

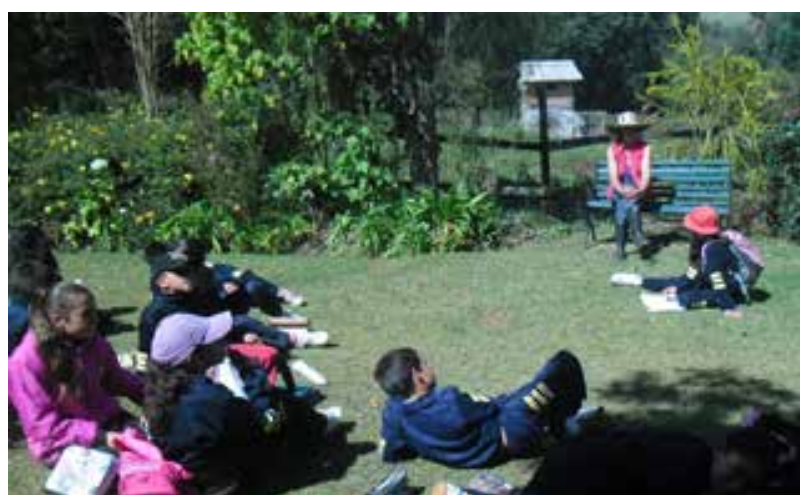

Para recordar los usos y significados culturales que se están perdiendo en las familias de los estudiantes, desde la práctica pedagógica se plantea una ruta metodológica ${ }^{5}$ para la renovación de la memoria histórica desde la cultura del maíz. Para esto se tomó como referente el trabajo del pueblo inga, en su modelo pedagógico.

Esta ruta pedagógica implica: desenvolver la palabra con los mayores para recordar las historias asociadas al maíz; los estudiantes dialogaron con sus abuelos y padres sobre refranes, adivinanzas y otras narrativas vinculadas con el maíz, y realizaron un registro en textos escritos y dibujados.

Para esto se recorrió el territorio con los estudiantes y sus padres, y se adelantaron visitas a sus fincas en sus trabajos diarios, con el propósito de conocer las casas cercanas

5 Rutas pedagógicas propuestas por el modelo Etnoeducativo: El planteamiento y desarrollo de la ruta pedagógica no es lineal, es decir los diferentes momentos pueden converger, ser, paralelos, desarrollarse permanentemente desde todos los ciclos. Metodológicamente se propone que posterior al desarrollo de las diferentes actividades de las rutas pedagógicas, hayan espacios de socialización y retroalimentación comunitaria sobre lo aprehendido, dudas, comentarios, proyecciones, a través de mingakuna, exposiciones, juegos, obras de teatro, realización de videos, conversatorios, entre otros.

También se debe dejar memoria textual, entendida como tejido de significados que se expresan en diferentes lenguajes: escritos, videos, arte. 
de algunos de los estudiantes y dialogar con sus familiares acerca de la cultura del maíz; se les interrogó sobre recetas gastronómicas vigentes, y si aún cultivan este producto.

Esta salida de campo finalizó con una socialización y retroalimentación, a través de una actividad de cartografía social ${ }^{6}$, para comprender el territorio y construir conocimiento colectivo frente al tema,

Se identificó prácticas en torno al maíz, cultivando con técnicas tradicionales, en la huerta escolar en donde los hombres prepararon la tierra desyerbando, arando, haciendo los surcos y los huecos mientras las mujeres ponían la semilla.

Además, para guardar memoria con textos de imagen y escritos en una bitácora de las historias asociadas al maíz, algunos refranes, adivinanzas, las recetas gastronómicas que nos dieron las familias de los estudiantes, la técnica tradicional de cultivar y las etapas del crecimiento y desarrollo de la planta del maíz, complementando este trabajo se realizó un video con las conversaciones e imágenes que se obtuvieron durante la salida de campo, la siembra y demás actividades.

\section{Resultados y discusión}

Para el análisis de resultados se realiza una matriz de contingencia en donde se tomaron en cuenta cinco aspectos como categorías para su análisis; la primera de ellas es historias asociadas al maíz, en la cual se encontró que solo una estudiante conoce una de ellas, y además cuenta cómo los indígenas preparaban chicha.

\section{El arte de los chibchas con el maíz}

Unos de los que sembraba maíz eran los chibchas; ellos lo cultivaban y lo molían con los dientes y también lo trituraban con grandes rocas y luego de eso lo almacenaban en grande ollas de barro y lo utilizaban para hacer sus chichas y sus mazamorras de maíz. (Dayanna Alayon).

6 La cartografía social es una herramienta que sirve para construir conocimiento de manera colectiva; es un acercamiento de la comunidad a su espacio geográfico, social, económico, histórico y cultural. La construcción de este conocimiento se logra a través de la elaboración colectiva de mapas, la cual desata procesos de comunicación entre los participantes y pone en evidencia diferentes tipos de saberes que se mezclan para poder llegar a una imagen colectiva del territorio (Tropenbos Internacional, Nuffic y SENA, 2009).

\section{El maíz}

Había una vez un señor que le gustaba sembrar maíz y ya había cogido todo el maíz, entonces volvió a sembrar maíz primero se abona la tierra con abono de cabra, etc., y se revuelve la tierra con el abono luego de hacer surcos con el palo de escoba luego se siembra el maíz y a veces la echan con una haba al lado. Después la tapa cuando está mediano se aporca y cuando está grande se quita el pasto que hay en el medio de los surcos con la pala y luego esta para coger y se coge y se puede hacer tamales. (Eduar Cruz).

\section{El sembrador de maíz}

Había una vez un señor que tenía un cultivo de maíz y cuando la cosecha estaba buena cogía el maíz e iba y lo vendía en el pueblo. Con la plata que recogía de la venta del maíz compró semilla y la sembró, hubo un vendaval y le dañó toda la cosecha. El señor se puso triste entonces pidió un préstamo al banco y compró más semilla de la que compraba antes; la sembró y tuvo buena cosecha le dio mucho maíz entonces, lo vendió y le llegó más dinero. Pagó la deuda al banco, vivió feliz y el señor siguió sembrando maíz mientras vivió. (Miguel Ángel Alfonso).

En esta categoría se pudo evidenciar que tanto los estudiantes como los padres de familia, en su mayoría, no conocen historias asociadas al maíz, esto porque cuando se hicieron las conversaciones y visitas a sus casas, no contaron ninguna historia o tal vez ya no la recuerdan.

La segunda categoría es cómo se siembra el maíz. De aquí se podría decir que todos los niños conocen las etapas de siembra y algunos, la labor de la mujer para poner la semilla. Este conocimiento fue adquirido mediante la observación, pues los niños acompañan a sus abuelos a sembrar, mientras las niñas están con sus abuelas preparando la comida y solo las Ilaman para poner las semillas "[...] nosotros con mi abuelita Hacíamos la comida. Y cuando nos llaman a echar la semilla vamos y la echamos y los hombres lo tapan con tierra y cada quien sale pasto toca desyerbar el maíz" (Karen Urrego).

Todos de los estudiantes realizaron dibujos en los que muestran los pasos para cultivar maíz y algunos reconocen la labor de la mujer.

La tercera categoría son las familias que siembran maíz. Aquí se pudo evidenciar durante las conversaciones y visitas, que en este momento ya no siembran, en su gran mayoría; esto se ha ido perdiendo con el paso de los años, algunos padres afirman que es por su largo periodo de crecimiento y porque no es rentable. 
Doña Graciela, abuela de Isabela, una de las estudiantes del grupo, dice:

[...] no... quieren que no toque trabajar, el maíz tienen que desyerbarlo, aportarlo y nooo porque ni da el producto, tiene costos, cuando siembran un lote de zanahoria y venden millonadas. Pero aquí sí, aquí se siembra en la huertica, aquí está naciendo uno y va nacer otro.

Durante las conversaciones se puede evidenciar cómo se han ido desplazando estas costumbres y tradiciones que antes los abuelos de los estudiantes tenían como en el caso de la familia de Juliana. Su mamá comenta que sus padres antes sí sembraban harto maíz para el consumo de ellos, pero ahora no.

La cuarta categoría son las recetas gastronómicas. Las niñas tienen más claro cómo se preparan las recetas con maíz a comparación de los niños que la han comido pero no saben cómo prepararla, porque las mujeres son las encargadas de cocinar.

Durante el recorrido y visitas en la salida de campo, la señora Graciela y la mamá de Juliana mencionaron varias recetas con maíz.

Refranes o adivinanzas es la última categoría en donde solo Vanessa, Juliana y Yuri conocen adivinanzas y refranes del maíz.
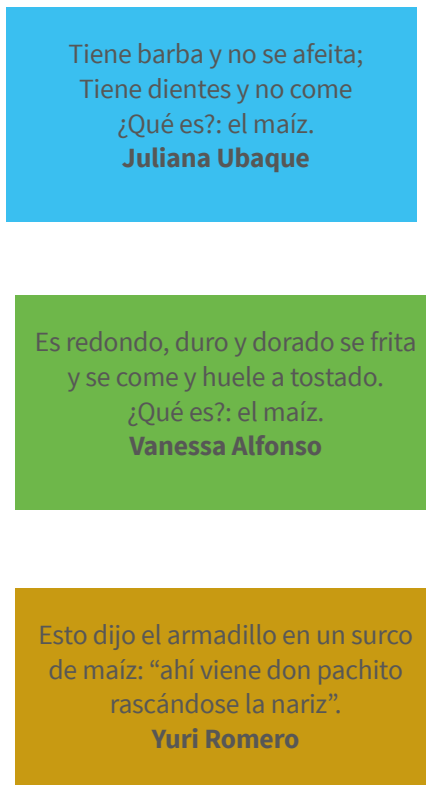

Además se analizaron otras actividades como la socialización y retroalimentación, lo cual se realizó luego de terminar el recorrido por el territorio, en la salida de campo, mediante la cartografía social. Posteriormente se elaboró un mapa del territorio, se trazaron los recorridos de las casa que se visitaron y se estableció la relación con el cultivo de maíz; de esta manera se logró identificar que hay familias que aún siembran maíz. Para ello se organizaron dos grupos desde los cuales se reflexionó y se representó la realidad mediante símbolos o dibujos, para finalmente exponerlos y guardar memoria (Foto 1).

Por otro lado, la siembra de maíz en la huerta escolar fue dirigida y ejecutada por los estudiantes, quienes recordaron, paso a paso, cómo se sembraba maíz con técnicas tradicionales: primero unos niños se encargaron de desyerbar, luego revolvieron la tierra con el abono de estiércol de vaca; posteriormente tres niños cada uno con sus herramientas (azadón) se encargaron de hacer los surcos, después hicieron unos huecos con un palo; en cada uno de estos, las mujeres y las niñas colocaron tres semillas de maíz para, finalmente, taparlos. Cabe aclarar que este procedimiento fue aprendido tras observar a sus familiares padres, abuelos, etc. trabajar en el campo.

En esta actividad se evidenciaron las prácticas que los campesinos de esta región tienen en torno al cultivo de maíz y la importancia de las mujeres en la siembra al poner la semilla, relacionándolo con la fertilidad de la mujer para la tierra. Es importante también mencionar que solo las mujeres son las encargadas de preparar la comida, y son quienes arreglan la tierra para sembrar.

\section{Conclusiones}

Durante la investigación y la interacción con la comunidad educativa de El Carmen sede San Francisco, en Guasca, se conocieron sus tradiciones, costumbres, formas de vida y el quehacer campesino. Esto evidencia que los estudiantes mantienen un diálogo con su cultura y su ambiente natural, lo que Toledo y Barrera (2008) Ilaman memoria biocultural.

Los estudiantes poseen saberes que han sido trasmitidos por sus familiares padres, abuelos, etc. de generación en generación, como abuelos y padres; además desde la observación adquieren un conocimiento de la siembra de maíz, de sus preparaciones, de su historia, de la cultura del maíz, esto siendo posible por la memoria individual y colectiva de los habitantes de esta región.

Esta memoria individual hace posible renovar la memoria histórica sobre la cultura del maíz, trascendiendo a una producción de conocimiento colectivo y haciendo evidente las relaciones con la naturaleza y el territorio. 
El proceso de renovación de memoria se realizó desde las experiencias, los sentires y conocimientos trasmitidos de las familias de los estudiantes. Desde allí se evidenció que sus tradiciones se pueden rememorary recuperar las que se están perdiendo por medio de la tradición oral, los usos y significados sobre la cultura del maíz.

Para la investigación, en principio, no se tenía un diseño previo de las actividades; pero a través de la interacción con la comunidad este fue adquiriendo forma; así surgió una ruta metodológica con unos propósitos pedagógicos, entre ellos: fortalecer las tradiciones y costumbres culturales de los estudiantes; reconocer la importancia de sembrar con técnicas tradicionales utilizando la huerta escolar, e incentivar los procesos de lectura y escritura con significado.

Estos procesos representan una forma de enseñar biología no desde un salón de clases, sino interactuando con su entorno y su relación con lo vivo desde un enfoque intercultural, en donde todos aprendemos en un diálogo de saberes, en el cual cada persona está en contacto permanente con el otro.

Finalmente desde lo que plantea Montes y Rodríguez (1975), la comunidad educativa posee una cultura del maíz ya que se pudo recoger desde los textos de imagen y escritos de los estudiantes, una recopilación de adivinanzas, refranes, historias, recetas gastronómicas, creencias y costumbres entorno al maíz que forman parte del vivir de los estudiantes.

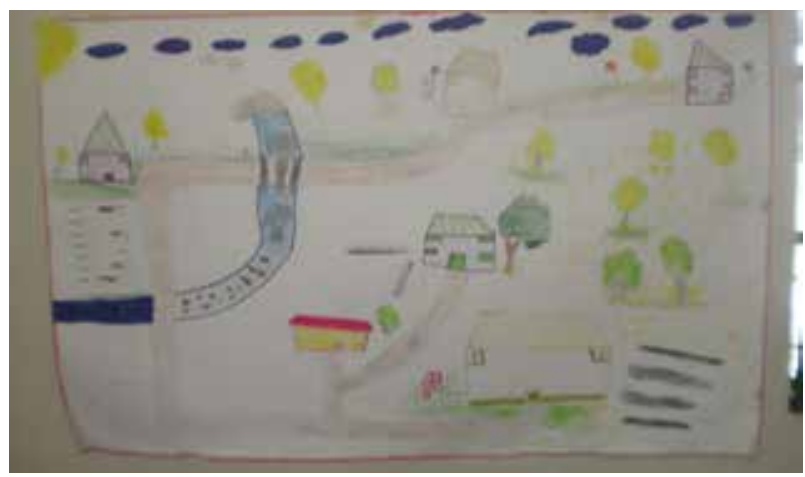

Foto 1. Cartografía realizada por los estudiantes, recorriendo nuestro territorio.

\section{REFERENCIAS BIBLIOGRÁFICAS}

Denzin, N.; Lincoln, Y. y Vonna, S. (2005). The Sage Handbook of Qualitative Research (Third Edition). Thousand Oaks: Sage Publications Inc.

Lloréns, J. (2008). Chicha: De la bebida a la cultura. Perú: Fondo Editorial Universidad de San Martin de Porres.

Ministerio de Cultura (s.f.). Prosperidad para todos. Recuperado el 5 de junio de 2013 de: http://www. mincultura.gov.co/?idcategoria $=41800 \#$

Montes, J. y Rodríguez, M. (1975). El maíz en el habla y la cultura popular de Colombia: Notas sobre su origen y nombres en lenguas indígenas americanas. Bogotá: Publicaciones Instituto Caro y Cuervo XXXIII.

Pueblo Inga. (2011). Guía de actividades específicas: Mama Sara-Madre Maíz o del alimento, la más importante de las conopas (representaciones religiosas) de los alimentos junto con la coca y papa. Caquetá: Inédito.

Reinoso, E. (2012). Cuaderno de campo de práctica pedagógica.

Toledo, V. y Barrera, N. (2008). la memoria biocultural, la importancia ecológica de las sabidurías tradicionales. Barcelona: Icaria Editorial.

Tropenbos Internacional, Nuffic y Servicio Nacional de Aprendizaje (SENA). (2009). Formación en gestión ambiental y cadenas productivas sostenibles, herramientas de trabajo en cartografía social. Recuperado el 25 de mayo de 2014 de: http://tropenbos. sena.edu.co/DOCUMENTOS/HERRAMIENTAS\%20 METODOLOGICAS/5\%20Guia_cartografia_social.pdf 